\title{
Implantable device procedures and introduction of ablation in University Hospital Dubrava before and during the COVID-19 pandemic
}

\author{
(iD)Ante Lisičić, \\ DSanda Sokol Tomić, \\ Nikša Bušić, \\ (D)Petra Vitlov, \\ (DIrzal Hadžibegović *
}

University Hospital Dubrava, Zagreb, Croatia

\author{
KEYWORDS: electrostimulation, cardiac resynchronization, ablation. \\ CITATION: Cardiol Croat. 2021;16(1-2):11. | https://doi.org/10.15836/ccar2021.11
}

*ADDRESS FOR CORRESPONDENCE: Irzal Hadžibegović, Klinička bolnica Dubrava, Avenija Gojka Šuška 6, HR-10000 Zagreb, Croatia. / Phone: +385-91-5333091 / E-mail: irzalh@gmail.com

ORCID: Ante Lisičić, https://orcid.org/0000-0002-4365-9652 • Sanda Sokol Tomić, https://orcid.org/0000-0002-4551-9231 Petra Vitlov, https://orcid.org/0000-0001-6983-1409 • Irzal Hadžibegović, https://orcid.org/0000-0002-3768-9134

|IIIIIIIIIIIIIIIIIIIIIIIIIIIIIIIIIIIIIIIIIIIIIIIIIIIIIIIIIIIIIIIIIIIIIIIIIIIIIIIIIIIIIIIIIIIIIIIIIIIIIIIIIIIIIIIIIIII

Background: Case of "missing patients" with coronary artery disease and myocardial infarction during the COVID-19 pandemic is widespread. Implantable devices procedures and ablation procedures have also been affected, however reports are few. ${ }^{1}$ Aim: To compare the numbers of implantable cardiac devices procedures between 2019 and 2020.

Patients and Methods: Numbers and complications of single-chamber (VVI) and dual-chamber pacemakers (DDD), implantable cardiac defibrillators (ICD), and cardiac resynchronization devices (CRT) implantations were analyzed and compared between the period from Jan 2019 to Dec 2019 and Jan 2020 to Dec 2020.

Results: In a 12-month operating period in 2019, there were 88 VVI, 103 DDD, 25 ICD, and 16 CRT implantations. In comparison, during a 7-months working period in 2020, there were 53 VVI, 88 DDD, 36 ICD, and 15 CRT implantations. The remaining 5 months of 2020 we worked as a COVID-19 dedicated hospital with no non-urgent and elective procedures, and performed only 1 urgent VVI and 1 urgent DDD implantation in COVID-19 positive patients. In addition, invasive electrophysiology and ablation of cardiac arrhythmias has been introduced in December 2019, with 33 invasive EP procedures with ablation performed by the sole interventional electrophysiologist in 2020. There were $15 \mathrm{AF}$ ablations using cryoballoon technology, 6 SVT ablations (focal AT and AVNRT) using 3D electroanatomical mapping system (4 of which were "zero fluoro" procedures), and the rest were miscellaneous SVT ablations (mostly AVNRT and CTI dependent atrial flutter) using conventional approach. There were no differences in complications or duration of hospitalization between the two periods.

Conclusion: COVID-19 pandemic reduced the number of VVI and DDD implantations, that are more frequently urgent in the elderly population. However, in 2020, CRT and ICD implantations increased absolutely and relatively (respectively), and would probably increase even more without the pandemic situation, ensuring optimal treatment for more heart failure patients with appropriate indications. Also, we expected that the numbers of ablations would probably increase over time without the pandemic situation. However, there was probably a negative impact on patients' consent to be ablated in a COVID-19 dedicated hospital.
RECEIVED:

December 14, 2020

ACCEPTED:

December 18, 2020

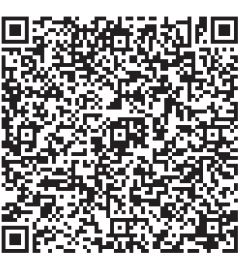

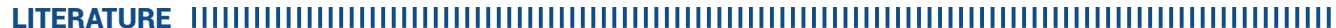

1. Marini M, Zilio F, Martin M, Strazzanti M, Quintarelli S, Guarracini F, Coser A, Giacopelli D, Bonmassari R. COVID-19 pandemic and elderly: is the curtain dropped for urgent pacemaker implantations? Minerva Cardioangiol. 2020 Dec 1.

https://doi.org/10.23736/S0026-4725.20.05451-1. Epub ahead of print. 